\title{
Research on the Generalized Decision Reducts and the Acquisition of Optimal Decision Rules in Generalized Decision Information System
}

\author{
Chen Keming ${ }^{1,2}$, Zheng Jian-guo ${ }^{2}$, Li Kang ${ }^{3}$ and Deng $\mathrm{Li}^{4}$ \\ ${ }^{1}$ School of Business and Management, Donghua University, Shanghai 200051, China; ${ }^{2}$ School of Continuing Education, \\ XinYu University, XinYu University, JiangXi, 338004, China
}

\begin{abstract}
This paper discusses generalized decision reducts and the acquisition of optimal decision rules in general (consistent or inconsistent) decision information systems. The properties and relationship between Pawlak reducts with respect to definite information and generalized decision reducts based on indefinite information were analysed and revealed respectively. Secondly, the computation approach of reducts and optimal decision rules based on discernibility functions were given. Last but not the least, a type of improved discernibility matrix was constructed, which can be used to compute generalized decision reducts and generalized decision core as well as Pawlak reducts and core. The discussions in this paper considered the consistency of information systems, in making a distinction between Pawlak reducts and generalized reducts. This work improved and generalized the relevant study by senior researchers. Moreover, some misunderstandings in the computation of cores in information systems have also been clarified from mathematical point of view.
\end{abstract}

Keywords: Acquisition of optimal decision rules, generalized decision information system, pawlak reducts, the generalized decision reducts.

\section{INTRODUCTION}

Rough set theory was proposed by Professor Z. Pawalak in 1982. It is an effective tool to deal with uncertainty and incomplete information, and used it successfully in machine learning, data mining, decision analysis, inductive reasoning, and so on. Based on the mechanism of classification, rough set theory considers the classification of universe as a form of knowledge. This idea is the main and important thought of rough set theory. Comparing with other mathematic tools which are used for handling uncertainty and vague problems, such as probability theory, fuzzy theory and evidence theory, rough set theory has its own outstanding advantages. It does not need subjective information beside the known objective data, and describes and resolves the uncertainty problems objectively.

The researches on the mathematical theory of classical rough set, including axiom problem, topology structure, algebraic structure and so on, have achieved fruitful results [1, 2]. Y.Y. Yao discussed constructive and algebraic methods of rough set theory, and studied the relationship between rough set and other mathematic concepts such as pairs of definable set, interval set, families of subsets and rough member function from set-oriented view, interior and closure operator in topological space, necessity and possibility operators in modal logics, and lower and upper approximations in interval structure from operator-oriented view [3, 4]. There are three key factors relative to classical rough set, they are; universe, binary relation on the universe and categories. Therefore, the classical rough set model can be extended according to these three factors.

*Address correspondence to this author at the School of Business and Management, Donghua University, Shanghai 200051, China; Tel: 13879068233; E-mail: 403848072@
In addition, by generalizing classical inclusion relation to approximate inclusion relation, classical rough set model can be extended further. The binary relation in classical rough set is equivalent. That is, it is reflexive, symmetric and transitive. By removing or replacing the properties of the equivalent relation in classical rough set model, some different extensive models can be obtained [4]. For example, generalized approximate space in which approximate operators are neighbourhood operators determined by an arbitrary binary relation [5, 6]; generalized approximate space in which the binary relation is reflexive and transitive [7]; generalized approximate space in which the binary relation is reflexive, anti-symmetric and transitive [8,9]; and tolerant rough set model in which the binary relation is reflexive and symmetric. In addition, by generalizing the classical binary relation to fuzzy relation, fuzzy approximate space can be obtained [10].

In classical rough set model, for the relationship between two subsets of universe, only the terms "include" and "don't include" are considered. Sometimes this idea has its own restriction, because "include" and "don't include" are only two special cases; they cannot describe the approximate inclusion (inclusion to some extent), and this may lead to information loss in practice. In order to resolve this problem, two extended rough set models are introduced: (1) variable precision rough set model; (2) rough set model based on uncertainty function and rough inclusion function, which is a generalization of classical rough set model, variable precision rough set model and generalized rough set model based on reflex relation.

\section{ROUGH SET THEORY WITH OTHER MATHE- MATIC THEORIES}

Fuzzy set and rough set both are generalizations of classical set. In view of "knowledge granularity", fuzzy set de- 
scribes the degree of an object belonging to one set by so called membership function. It emphasizes vagueness of boundary of the set; whereas, based on the classification of universe by indiscernibility relations, rough sets theory uses lower and upper approximation to describe the set, emphasizing the indiscernibility of objects. Fuzzy sets describe the degree of objects belonging to the same class; whereas rough sets describe the relationship of an object with its indiscernibility class to other sets. Both membership functions of fuzzy sets and rough membership functions of rough sets represent the uncertainty of concepts, but membership functions of fuzzy sets are always given by experts subjectively, whereas rough membership functions of rough sets can be obtained directly from data.

D. Dubois and H. Prade considered the combination of fuzzy set and rough set, and proposed fuzzy rough set and rough fuzzy set. Other works on fuzzy rough set and rough fuzzy set can be seen in related references. Knowledge discovery in data base (KDD) is one of the important applications of rough set theory. So far, several RS data mining systems have been developed, such as LERS of Kansas university in America, Rough DAS and Rough Class of Poznan technology university in Poland, KDD-R of Reginain University in Canada. Attribute reduct and optimal decision rules acquisition in information systems have been important research fields.

The main applications of rough sets theory to information systems are attribute redact and decision rules optimization. In information systems, all the attributes are not necessary. By attribute redact, under a certain standard, it can be decided which attributes are absolutely necessary, which attributes are relatively necessary and which attributes are abundant. Thus, it is important to evaluate the importance of attributes, and redact abundant attributes. Attribute redacts are clarsfied into redacts of system and redacts of decision rules. The redact of the system is an attributes' set which keeps a certain information of the original system. The redact of a decision rule, which is called redact of object or also value redact, is a conditional attributes' subset which determines the decision values. Finding the redacts of a decision rule and deleting the abundant conditional attributes, optimal decision rules can be derived. Discernibility matrix approach and information approach are two important methods for computing attribute redacts. This paper utilizes discernibility approach to compute attribute redacts and make optimal decision rules in complete decision information systems (decision tables). Pawlak proposed the relative redact of decision tables [11, 12] which keeps the relative positive field unvaried. This kind of redacts concerns only definite information of the system, and does not consider indefinite information. Thereafter, many concepts of attribute redacts have been proposed according to different purposes, such as possible redacts, approximate redacts, generalized decision redacts, Udecision redacts are equivalent to u-redacts (known as quantitative redacts); and quantitative redact must also be qualitative redact. Both qualitative redacts and quantitative redacts are redacts from algebraic viewpoint. Distribute redact and possible redact proposed by Zhang et al. in Ref. [13] coincide with the $\mu$-decision redact.
The qualitative reduct of decision table is defined by the reducts of objects. The reducts of objects induce the corresponding optimal decision rules. Kryszkiewicz discussed in Ref. [14] the relationship of possible reducts, approximate reducts, generalized decision reducts, /.c-decision reducts, and $\mu$-reducts. Liang and $\mathrm{Li}$ improved in Ref. [15] the results of Kryszkiewicz, and pointed out that the relationships between the reducts of the system are different with the relationships between reducts of the object.

The reducts of decision table are not necessarily the reducts of objects, therefore, they may not induce optimal decision rules. In fact, the reducts of decision table are minimal conditional attribute sets which can simplify all the decision rules. Discernibility matrix $[16,17]$ proposed by Skowron and its improved forms proposed by others, are effective tools for computing reducts of decision table. In order to compute the core attributes of decision table, Hu and Cercone proposed in Ref. [18] a kind of improved discernibility matrix (called Hu-improved discernibility matrix in this section) and Ye and Chen pointed out in Ref. [19] the drawback of Hu-improved discernibility matrix, and gave other improved discernibility matrix (called Yu-improved discernibility matrix in this section). Wang pointed out in Ref. [20] that it is the inconsistency of the decision table that results in the creating error in Hu-improved discernibility-matrix. Wang compared the cores of system computed by Hu's and Ye's discernibility matrices with the core of system under information viewpoint. The discussion in this section confirms that Hu's and Ye's discernibility matrices did not concern the difference in the consistency and inconsistency of the system, and either generalized decision reducts.

This paper discusses the generalized decision reducts of inconsistent decision table, proposes an improved discernibility matrix, and compares it with Hu's and Ye's discernibility matrices. It can be seen that Hu's, Ye's and the definitions of discernibility matrix discussed in this paper coincide with each other for consistent decision tables. For inconsistent decision tables, Hu's discernibility matrix would be invalid, Ye's discernibility matrix can be used to compute Pawlak reduct only, whereas the discernibility matrix proposed in this paper can not only be used to compute generalized decision reducts, but also can be used to compute Pawlak reducts by its sub-matrix corresponding to consistent objects.

\section{ATTRIBUTE REDACTS AND OPTIMAL DECISION RULES IN INFORMATION SYSTEMS}

An information system is a quadruple $\mathrm{S}=\langle\mathrm{U}, \mathrm{A}, \mathrm{V}, \mathrm{f}\rangle$, where $U$ is a non-empty finite set of objects, $A$ is an attributes set $V=\cup V_{a}$, where $\mathrm{V}_{\mathrm{a}}$ is the domain of a, $a \in A$; $f: U \times A \longrightarrow \stackrel{a \in f}{\longrightarrow}$ is an information function such that for $x \in U$ and $a \in A, f(x, a) \in V_{a}$. Denoting $\mathrm{f}(\mathrm{x}, \mathrm{a})=\mathrm{a}(\mathrm{x})$ for convenience.

If each object has unique known attribute value for every attributes in information system $\mathrm{S}=<\mathrm{U}, \mathrm{A}, \mathrm{V}, \mathrm{f}>\mathrm{S}=<\mathrm{U}, \mathrm{A}$, $\mathrm{V}, \mathrm{f}>$ is known as complete information system.

In this system, complete information is clarified to condition system $\mathrm{S}=<\mathrm{U}, \mathrm{A}, \mathrm{V}, \mathrm{j}>$, attributes and decision if the 
attributes in A are, $A=C \cup D, C \cap D=\varnothing$, we call $\mathrm{S}=<\mathrm{U}, \mathrm{C}$ $\cup \mathrm{D}, \mathrm{v}, \mathrm{f}>$ decision information system. It is always represented as a table which is called the decision table. For convenience, we assume that $\mathrm{D}=\{\mathrm{d}\}$. Let $\mathrm{S}=<\mathrm{U}, \mathrm{C} \cup\{\mathrm{d}\}, \mathrm{V}, \mathrm{f}>$ be a decision information system, and $\mathrm{B} \subseteq \mathrm{C}$. Defining an indiscernibility relation IND(B) on $\mathrm{U}$ is as follows:

$$
\operatorname{IND}(B)=\{<x, y>\mid<x, y>\in U \times U,
$$$$
\forall b \in B, b(x)=b(y)\}
$$

The equivalent class containing object $\mathrm{x}$ is denoted by $[\mathrm{x}]_{\mathrm{IND}(\mathrm{B})}$ or $[\mathrm{x}]_{(\mathrm{B}) .} \mathrm{X} \subseteq \mathrm{U}$, denoting the lower and upper approximations of $\mathrm{X}$ with respect to $\mathrm{IND}(\mathrm{B})$ as follows:

$$
\begin{aligned}
& \underline{B}(X)=\cup\left\{[x]_{B} \mid[x]_{B} \subseteq X\right\} \\
& \bar{B}(X)=\cup\left\{[x]_{B} \mid[x]_{B} \cap X \neq \varnothing\right\}
\end{aligned}
$$

and the relative positive field of $\mathrm{B}$ is denoted with respect to d as:

$\operatorname{POS}_{B}(\mathrm{~d})=\cup\left\{\underline{B}\left(D_{i}\right) \mid D_{i} \in U / I N D(d)\right\}$

It is the set of objects which can be clarified definitely to decision equivalent classes according to classified information of attributes set B.

Definition 1: Let

$\gamma_{B}(d)=\sum_{i=1}^{k} \frac{\left|\underline{B}\left(D_{i}\right)\right|}{U}=\frac{\left|\operatorname{POS}_{B}(d)\right|}{U}$

$\gamma_{B}(d)$ is called as B-approximate precision of partition $\left\{\mathrm{D}_{1}, \mathrm{D}_{2}, \ldots, \mathrm{D}_{\mathrm{k}}\right\}$.

The set of generalized decision reducts of $\mathrm{x}$ is denoted as $\operatorname{red}(\mathrm{x})$, call the intersection of all generalized decision redact of $\mathrm{x}$ as generalized decision core of $\mathrm{x}$ and denoted it as $\operatorname{core}(\mathrm{x}, \mathrm{C})$.

The optimization of decision rules is often defined as a process of simplifying the description of condition attributes of rules under the same decision to make conclusions. In this way, the necessary condition attributes for decision conclusions could be found.

Definition 2: If $\mathrm{B}(\mathrm{B} \subseteq \mathrm{C})$ is a minimal attribute set such that $\partial_{B}(x)=\partial_{C}(x)$ for $\forall x \in U$, where $\mathrm{B}$ represents a generalized decision reduct of decision.Information system calls for the intersection of all generalized decision redacts as generalized decision core of decision information system, which is denoted by G-core.

The generalized decision redact of information system is a minimal conditional attributes set which simplifies all the decision rules, but it is not necessarily a redact of certain decision rules. The relationship between redacts of system and redacts of objects can be seen clearly from the discernibility function defined below.

Let $\mathrm{B} \subseteq \mathrm{C}$, then the following expressions (1)-(4) are equivalent with each other:

(1) B is a Pawlak reduct of decision information system.

(2) $\mathrm{B}$ is a minimal condition attributes set satisfying $\operatorname{POSE}_{\mathrm{B}}(\mathrm{d})=\operatorname{POSE}_{\mathrm{C}}(\mathrm{d})$.
(3) $\mathrm{B}$ is a minimal conditional attributes set satisfying $\underline{B}\left(D_{i}\right)=\underline{C}\left(D_{i}\right)$ for $\forall D_{i} \in U / \operatorname{IND}(d)$.

(4) $\mathrm{B}$ is a minimal conditional attributes set satisfying $\partial_{B}(x)=\partial_{C}(x)$ for $\forall x \in C(U)$.

The possible reducts and approximate reducts are equivalent to generalized decision reducts, therefore, they can be viewed as the equivalent description of generalized decision reducts. Another equivalent description of the generalized decision reduct is shown as below:

$\mathrm{B}$ is a generalized decision reduct of decision information system $\Leftrightarrow \mathrm{B}$ is a minimal condition attributes set satisfying $\underline{B}\left(D_{i}\right)=\underline{C}\left(D_{i}\right)$ for $\forall D_{i} \in U / I N D(d)$.

Pawlak reducts merely simplify definite decision rules, and it cannot simplify all the indefinite decision rules. But generalized decision reducts can simplify all the decision rules.

Example 1: will illustrate approaches to compute reducts, core, and optimal decision rules for the inconsistent decision table presented in Table 1.

Table 1. An inconsistent decision table.

\begin{tabular}{|c|c|c|c|c|c|}
\hline \multirow{2}{*}{$\mathrm{U}$} & \multicolumn{5}{|c|}{ Factors } \\
\cline { 2 - 6 } & $\mathbf{C 1}$ & $\mathbf{C 2}$ & $\mathbf{C 3}$ & $\mathbf{C 4}$ & $\mathbf{d}$ \\
\hline \hline $\mathrm{X} 1$ & 1 & 0 & 0 & 0 & 1 \\
\hline $\mathrm{X} 2$ & 0 & 1 & 1 & 1 & 2 \\
\hline $\mathrm{X} 3$ & 1 & 1 & 0 & 0 & 2 \\
\hline $\mathrm{X} 4$ & 0 & 1 & 1 & 0 & 3 \\
\hline $\mathrm{X} 5$ & 1 & 1 & 0 & 0 & 1 \\
\hline $\mathrm{X} 6$ & 1 & 0 & 0 & 0 & 3 \\
\hline $\mathrm{X} 7$ & 1 & 1 & 1 & 1 & 2 \\
\hline
\end{tabular}

Example 2: Table 2 is an inconsistent decision table.

Table 2. An inconsistent decision table.

\begin{tabular}{|c|c|c|c|c|}
\hline \multirow{2}{*}{$\mathrm{U}$} & \multicolumn{4}{|c|}{ Factors } \\
\cline { 2 - 5 } & $\mathbf{C 1}$ & $\mathbf{C 2}$ & $\mathbf{C 3}$ & $\mathbf{d}$ \\
\hline \hline $\mathrm{X} 1$ & 1 & 0 & 1 & 1 \\
\hline $\mathrm{X} 2$ & 1 & 0 & 1 & 0 \\
\hline $\mathrm{X} 3$ & 0 & 0 & 1 & 1 \\
\hline $\mathrm{X} 4$ & 0 & 0 & 1 & 0 \\
\hline $\mathrm{X} 5$ & 1 & 1 & 1 & 1 \\
\hline
\end{tabular}

This section discusses the properties of Pawlak reducts and generalized decision reducts and their connection, and proposes the computation approach of reducts of decision 
rules and systems by using Boolean reasoning techniques. A new definition of discernibility matrix, which can be used to compute Pawlak reducts and core, generalized decision reducts and core, and optimal decision rules was proposed. It was observed that this definition of discernibility matrix coincides with those of Hu's and Ye's for consistent decision tables, and for inconsistent decision tables. Moreover, Hu's indiscernibility matrix is invalid to compute Pawlak reducts and core, and generalized decision reducts and core, however, Ye's indiscernibility matrix can only be used to compute Pawlak reducts and core. In other words, the new definition of indiscernibility matrix can be viewed as the generalization of Hu's and Ye's definitions.

Wang and some other authors discussed the relationship between reducts of decision table from algebraic point of view and information. They found that, for consistent decision tables, definitions of reducts under these two views coincide with each other, but for inconsistent decision tables, they are not equivalent. It may be interesting to consider the relationship between the generalized reduct from algebraic point of view and information.

\section{TOLERANCE ROUGH SET MODELS AND IN- COMPLETE INFORMATION SYSTEMS}

By expanding the equivalent relation to more general binary relations, classical rough set model can be further generalized as general models. Approximate operators defined by neighbourhood operators based on arbitrary binary relation were studied. Approximate operators based on flexible and transitive relation were discussed in Ref. [13]. Approximate operators based on flexible relation were studied in Ref. [14]. Approximate space based on flexible and symmetric relation was discussed in Reference $[15,16]$. A general approximate space defined by uncertain function and rough inclusion function was discussed in Ref. $[17,18]$.

In practice, tolerant relation and partial order relation are two important binary relations. Approximate operator model based on partial order relation was proposed in Ref. [19], and successfully applied for the evaluation of risks involved. Tolerant rough set model was used to discuss attribute reduct and decision rule optimization in Ref. [20].

Some tolerant classes of the tolerant relation on a universe can form a cover of the universe, and tolerant rough set model can be derived by defining lower and upper operators using tolerant classes. Whereas, one tolerant relation on the universe may generate more than one cover, and this leads to inconvenient practice. In Ref. [21], the set of objects which are tolerant with a certain object is viewed as a class, and all such classes form a cover of universe. Unfortunately, objects contained in such a class may not be tolerant with each other, so they may not possess the common attribute description, and this leads to inconvenience for drawing decision rules. Therefore, the classification approach presented in Ref. [22] has some drawbacks. The cover of the universe consisting of maximal tolerant classes is determined uniquely by the tolerant relation. The maximal tolerant class is a maximal set of objects which possess a certain attribute description. Between two different maximal tolerant classes, there are no inclusion relations. Therefore, maximal tolerant classes can be viewed as neighbourhood and used to define lower and upper operators in this section.

In mathematical forms, complete information systems are special case of incomplete information systems containing null attribute values; incomplete information systems containing null attribute values are special case of incomplete information systems containing some objects with partial known attribute values and they are special set-valued information systems.

In complete information systems, each object has unique known attribute value with respect to every attribute. Therefore, we can define a discernibility relation on the universe, lower and upper operators by indiscernibility classes, and discuss attribute reduct and decision rules optimization. In incomplete information systems, attribute values of some objects with respect to certain attributes are unknown or partially known, and in set-valued information systems, objects may have multiple attribute values with respect to certain attributes, making it difficult to define an equivalent relation. On one hand, incomplete information systems can be transfixed to complete information systems, but this may result in informationloss. On the other hand, tolerant rough set model can be used to deal with incomplete information systems. This section gives new approaches using tolerant rough set model to discuss attribute reduct and optimal decision rules in incomplete information systems and set-valued information systems.

Two kinds of lower and upper approximate operators are defined by using maximal tolerant classes, respectively. This study analysed incomplete information systems containing null attribute values. Y-descriptor of the maximal tolerant class was proposed, and used to define the decision rules. Moreover, the concept of relative reduct of the maximal tolerant class and optimal credible decision rules were proposed, and a kind of corresponding discernibility function was defined. By computing minimal disjunctive forms of discernibility function of maximal tolerant classes, optimal credible decision rules can be derived. Attribute descriptors were introduced to define the lower and upper approximate operators, and the concept of reduct of the attribute descriptor was also proposed, but its computation approach was not given. Incomplete information systems containing objects with partial known attribute values were studied by attribute descriptors. In this section, a new kind of lower and upper approximate operator were defined, abd discernibility functions of attribute descriptors were designed to compute the reducts of attribute descriptors and induce optimal decision rules. The so called GS-reduct, DS-reduct, G-reduct and D-reduct were also defined to evaluate the significance of attributes.

Let

$R(x)=\{y \mid y R x, y \in U\}$
$R(x)=\cup\left\{K \mid K \in C C_{R}(x)\right\}$

For $X \subseteq U$, we have:

$I(X)=\left\{K \mid K \subseteq X, K \in C C_{R}(U)\right\}$ 
$O(X)=\left\{K \mid K \cap X=\phi, K \in C C_{R}(U)\right\}$

$$
B(X)=\left\{K \mid \phi \neq K \cap X \neq K, K \in C C_{R}(U)\right\}
$$

Then $C C_{R}(U)$ is the disjoint union of $I(X), B(X)$ and $O(X)$.

Definition : Let

$\underline{A R}(X)=\cup\left\{x \mid \forall K \in C C_{R}(x), K \subseteq X\right\}$

$\underline{E R}(X)=\cup\left\{x \mid \exists K \in C C_{R}(x), K \subseteq X\right\}$

Where, $\underline{A R}(X)$ and $\underline{E R}(X)$ are A-lower approximation and E-lower approximation of $X$ respectively; Let

$$
\begin{aligned}
& \overline{A R}(X)=\cup\left\{x \mid \forall K \in C C_{R}(x), K \cap X \neq \phi\right\} \\
& \overline{E R}(X)=\cup\left\{x \mid \exists K \in C C_{R}(x), K \cap X \neq \phi\right\}
\end{aligned}
$$

$\overline{A R}(X)$ and $\overline{E R}(X)$ represent the A-upper approximation and E-upper approximation of $X$ respectively; and $\underline{A R}(X), \underline{E R}(X), \overline{A R}(X)$ and $\overline{E R}(X)$ represent A-lower, Elower, A-upper E-upper approximate operator, respectively.

Both membership function of fuzzy set and rough membership function of rough set represent the vagueness of concepts. The membership function of fuzzy set is always given by experts subjectively, whereas the rough membership function of rough set is determined completely by analysed data, hence, it is objective. In approximate space, the equivalent classes are minimal recognition units, and the degree of an object belonging to a certain concept is determined completely by the relationship between the equivalent class containing the object and the concept, and in other words, it is determined completely by rough membership function. By using the rough membership function, various similarity degrees of rough sets were observed referring to the concepts of similarity degrees of fuzzy sets.

Both fuzzy sets and rough sets are generalizations of classical sets. This section summarizes the similarity measures of classical sets and fuzzy sets firstly. When fuzzy sets degenerate into classical sets, four kinds of ordinary fuzzy similarity degrees are degenerated into one of the three classical similarity degrees. In approximation space, because the objects contained in the same equivalent class are indiscernible, therefore, e the similarity degrees of rough sets are described using rough membership functions. In this study, eight kinds of rough similarity degrees referring to corresponding fuzzy similarity degree were proposed with demonstration of their properties. The properties of similarity degrees of classical sets, fuzzy sets and rough sets were also compared .

The main applications of rough sets theory on information systems include attribute redact and decision rules optimization. In information systems, not all the attributes are necessary. By attribute redact, a certain standard is set for determining all absolutely necessary attributes, relatively necessary attributes and abundant attributes. Thus, it is necessary to evaluate the importance of attributes, and redact abundant attributes. Attribute redacts are clasified into re- dacts of system and redacts of decision rules. The redact of the system is an attributes set which keeps a certain information of original system. The redact of a decision rule, which is called redact of object or also value redact, is a conditional attributes' subset which determines the decision values. Finding the redacts of a decision rule and deleting the abundant conditional attributes, optimal decision rules can be derived.

\section{CONCLUSION}

This paper, discussed the set-valued information systems. From mathematical point of view, set-valued information systems are generalization of complete and incomplete information systems. On the other hand, tolerant relation is generalization of equivalent relation. Maximal tolerant classification method can make objects contained in the same maximal tolerant class possess the same attribute description and objects possessing the same attribute description are in the same maximal tolerant class, hence the optimal decision rules can be computed conveniently. For this purpose, this study defined two kinds of lower and upper approximate operators using maximal tolerant classes, proposed three kinds of reduces/reducts of information systems to evaluate the significance of attributes; defined generalized and definite decision rules and their optimal decision rules, and proposed the concept of discernibility function of maximal tolerant class to compute optimal generalized decision rules.

Approaches used in previous papers are used to deal with incomplete information systems, the the approach discussed in section 4 can be used to deal with conjunctive interpreted set-valued information systems. Maximal tolerant classification method discussed in section 3 and 5 is used to make decision rules by classifying the universe. In this section, attributes descriptors in decision rules showing optimal decision rules are induced by discernibility function of descriptors.

\section{CONFLICT OF INTEREST}

The authors confirm that this article content has no conflict of interest.

\section{ACKNOWLEDGEMENTS}

Declared none.

\section{REFERENCES}

[1] H Sun. D.Y. Liu, and W. Li, "The minimization of axiom groups of rough sets," Chinese Journal of Computers, vol. 25, no.2, pp. 202-209, 2009.

[2] T.Y. Lin, "Neighborhood systems and relational database," In: Proceedings of CSC' 1988

[3] T. Y. Lin, and Y. Y. Yao, "Mining soft rules using rough sets and neighborhoods," In: Proceedings of the Symposium on I\}(odeting, Analysis and Simulation, Computational Engineering iw Systems Applications (CESA'96\}, IMASCS Multiconference, Lille, France, 1996, pp. 1095-1100.

[4] Y. Y. Yao, and Q. Liu, "A generalized decision logic in intervalset-valued information table," LNAI 1711, vol. 11, pp. 285-293, 1999.

[5] Y. Y. Yao, "Relational interpretation of neighborhood operators and rough set approximation operator," Information Sciences, vol. 111, pp. 239-259, 1998. 
[6] R. Slowinski, and D. Vanderpooten, "Similarity relation as a basis for rough approximations," In: P.P. Wang led., Advances in 'Machine Intelligence and Soft-Computing, Department of Electrical Engineering, Duke University, Durham, NC, USA, 1997, pp. 17-33.

[7] R.Slowinski, and D. Vanderpooten, "A Generalized.definition of rough approximations based on Similarity," IEEE Transactions on knowledge and Data Engineering, vol. 12, no.2, pp. 331-336, 2000.

[8] A. Skowron, and F. Stepaniuk, "Generalized approximation space, In: T. Y. Lin, A. M. Wildberger, (eds.), Soft Computing," Simulation Councils, San Diego, 1995, pp. 18-21.

[9] Y. Y. Yao, and S. K. M. Wong, "Generalization of rough sets using relation-ships between attribute values," In: Proceedings Second Annual 3oint Conference Information Sciences, pp. 30-33, 1995.

[10] Z. Bonikowski, E. Bryniarski, and U. W. Kardowska, "Extensions and intentions in the rough set theory," Information Science, vol.107, pp. 149-167, 1998

[11] W. Zhu, and F.Y.Wang, "Reduction and axiomization of covering generalized rough sets," Information Science, vol. 152, pp. 217$230,2003$.

[12] F. Zhu, and F.Y.Wang, "Some results about covering generalized rough sets," Chinese Journal of Pattern Recognition and Artif\}ciat Intelligent, vol. 15, pp. 6-13, 2002.

[13] S. Greco, B. Matarazzo, and R. Slowinski, "A new rough set approach to evaluation of bankruptcy risk. In: C. Zopounidis led.), Operational tools in the management of financial risks. Dordrecht, Kluwer
[14] M.A. Quinn, and S. Rubb, “Mexico's labor market: the importance of education-occupation matching on wages and productivity in developing countries," Economics Education Review, vol. 25, pp. 147-56, 2006.

[15] D.O. Rooth, and J. Saarela, "Selection in migration and return migration: Evidence from micro data," Economics Letters, vol. 94, pp. 90-95, 2007.

[16] W. Z. Wu, J. S. Mi, and W. X .Zhang, "Generalized fuzzy rough sets," Information Science, vol.151, pp. 263-282, 2003.

[17] W. Z. Wu, and W. X. Zhang, "Constructive and axiomatic approaches of fuzzy approximation operators," Information Science, vol. 159 , pp. 233-254, 2004.

[18] J.S. Mi, and W. X. Zhang, "An axiomatic characterization of a fuzzy generalization of rough sets," Information Science, vol. 160, pp. $235-249,2004$.

[19] A. T. Mahfoodh, and H. Radha, "Compression of image ensembles using tensor decomposition," In: Picture Coding Symposium (PCS) vol. 13, pp. 21-24, 2013

[20] L. Gan, "Block Compressed Sensing of Natural Images," In: The $15^{\text {th }}$ International Conference on Digital Signal Processing, pp. 403-406, 2007.

[21] Z. Jin, and G. Hu, "Application of inquiry optimized in the Distributed database system," Computer Applications and Software, pp. 58-60, 2013.

[22] B. Huang, X. He, and X. Z. Zhou, "Rough entropy covering redaction," Chinese Journal of Software based on generalized rough sets, vol.15, no.2, pp. 215-220, 2014.

Received: May 26, 2015

Revised: July 14, 2015

Accepted: August 10, 2015

(C) Keming et al.; Licensee Bentham Open

This is an open access article licensed under the terms of the (https://creativecommons.org/licenses/by/4.0/legalcode), which permits unrestricted, noncommercial use, distribution and reproduction in any medium, provided the work is properly cited. 